\title{
Transmission of Visual Data over Wireless Fading Channel in Real-time Systems Based on Superposition Coding Scheme.
}

\begin{abstract}
Real-time visual applications are among the most important requirements in the next generation wireless communication systems. In these applications, the transmitted data comprise different layers with different importance levels based on their influence on the reception quality. Furthermore, the real-time transmission nature of these applications makes them sensitive to data losses and transmission delay. To address these issues, an efficient superposition adaptive modulation and coding system, for the optimal system performance, is proposed in this paper. The proposed system switches its modulation and coding scheme adaptively to select the suitable modulation order and coding rate that best match with the instantaneous channel condition. The channel state information is estimated in receiver and fed back to transmitter. In such method, better performances in both data rate and bit error rate (BER) can be attained. Here, the source data are divided into different priority layers with different importance. Each layer bit stream is sent with specific error protection level against channel corruption. The highest error protection level is assigned to the highest priority layer, and vice versa. The modulated bit streams of all layers are then superimposed together and transmitted via Rayleigh fading channel. At the receiver side, a specific multistage decoding receiver is used to reconstruct the source data which demodulates the layers in the order of their priorities. Simulation results show that the proposed system provides up to $18 \mathrm{~dB}$ SNR and $46 \%$ data rate gains, respectively, compared to the traditional BPSK scheme at BER of $10-4$.
\end{abstract}

Keyword: Real-time wireless visual data transmission; Fading channel; Superposition coding; UEP; AMC 